\title{
Medicinal plants used to treat the most frequent diseases encountered in Ambalabe rural community, Eastern Madagascar
}

Nivo H. Rakotoarivelo ${ }^{1,2^{*}}$, Fortunat Rakotoarivony ${ }^{1}$, Aro Vonjy Ramarosandratana ${ }^{2}$, Vololoniaina H. Jeannoda ${ }^{2}$, Alyse R. Kuhlman ${ }^{3}$, Armand Randrianasolo ${ }^{4}$ and Rainer W. Bussmann ${ }^{4}$

\begin{abstract}
Background: Traditional medicine remains the only health care available in many rural areas in Madagascar like the rural community of Ambalabe, located in a very remote area in the eastern part of the country. With limited access to modern medicine, the local population uses medicinal plants to treat most diseases. In this study, we aimed to inventory medicinal plants used by local people and how those relate to the treatment of the most frequent diseases encountered in Ambalabe.

Methods: We interviewed participants in order to identify the most frequent diseases in the region and the medicinal plants used to treat them. The local physician was asked about the most frequent diseases, and ethnobotanical surveys to record medicinal plants and their uses, using semi-structured interviews and free listing, were conducted among 193 informants in local villages, of which $54 \%$ were men and $46 \%$ were women, ageing from 16 to 86 years. The local names, the uses of each plant species and the way they are prepared and administered were recorded and accompanied by herbarium specimens for identification. We also interviewed four traditional healers to elicit more details on the preparation and the use of plants.

Results: Our research allowed us to identify six most frequent diseases, namely diarrhea, malaria, stomach-ache, cough, bilharzia and dysentery. Among 209 plant species identified as having medicinal use, 83 species belonging to 49 families and 77 genera were used to treat these diseases. Our analyses highlighted the 11 commonly used species for their treatment, and also 16 species with a high fidelity level (FL $\geq 75 \%$ ) for each ailment. Diarrhea is one of the diseases with high number of species recorded.

Conclusions: This study highlighted the closed relationship between people in Ambalabe and plant species, especially when faced with frequent diseases. However, most of the species used were collected in the surroundings of the villages. Few species were from Vohibe forest in which a management system on the use of plant species was already established. Therefore, a sustainable use management should be considered for wild species from which medicinal plants are highly abundant.
\end{abstract}

Keywords: Medicinal plants, Madagascar, Ethnobotanical surveys, Frequent diseases, Conservation

\footnotetext{
* Correspondence: nivo.rakotoarivelo@mobot-mg.org

${ }^{1}$ Missouri Botanical Garden, Madagascar Research and Conservation Program,

BP 3391, Antananarivo 101, Madagascar

2Department of Plant Biology and Ecology, University of Antananarivo, BP

906, Antananarivo 101, Madagascar

Full list of author information is available at the end of the article
} 


\section{Background}

Traditional medicine has been used by the majority of the world population for thousands of years [1]. The World Health Organization (WHO) reported that an estimated $80 \%$ of the population in developing countries depend on traditionally used medicinal plants for their primary health care [2]. It is particularly the case in the rural and very remote area like the community of Ambalabe, in the Eastern part of Madagascar. In this area, sanitary conditions are very underdeveloped. A Basic Health Centre (Centre de Santé de Base or CSB) level II was established in the centre of the community (Ambalabe), with only a single doctor present 15 days per month. Thus, people resort to self-medication by buying drugs from peddlers, or prefer to use traditional medicine, which is often the only accessible and affordable remedy [3-5], and often associated with poverty [6].

People in Ambalabe community generally use plants for healing, and traditional healers are often consulted [7]. Medicinal plants are collected either in the surroundings of the villages, or in Vohibe forest which belongs to the community. Unfortunately, natural resources in Madagascar, including medicinal plants, are clearly affected by biodiversity loss, environmental degradation and a lack of sustainable harvesting practices [7-10]. These impacts are also exacerbated by climate change, and high levels of poverty [11].

Rapid deforestation and slash and burn cultivations (tavy) are threats that often affect medicinal plant habitat in the Eastern part of Madagascar [12], which may affect people's knowledge related to the use of medicinal plants. Furthermore, knowledge on these plants in Ambalabe community is still hardly documented at all. Only one paper addressed the issue on medicinal plants known by men [7], and knowledge erosion is currently observed worldwide [13, 14]. A lack of written documentation for Ambalabe community also adds to this problem, like shown in other countries [15]. Thus, this research was conducted with the aims to understand the importance of plant species as remedies, to document the knowledge on their uses among the local population especially when faced with frequent diseases, and to assess the degree of threats on those medicinal plants. To achieve our goals, we aimed to identify the most frequent diseases encountered in Ambalabe, and to inventory the medicinal plants used for their treatment and how they are used. Locations where these species were collected were recorded to find the number of species occurring in the local protected area. Our hypotheses were that (1) the local population has an important knowledge on plant species used to treat the most frequent diseases, and (2) most of medicinal plants are found in the surroundings of the villages and might be threatened by unsustainable collection and harvest practice. We focused on medicinal plants cited for the most frequent ailments and the area where they were collected.

\section{Methods}

The research was conducted with the contribution of the local staff of the Missouri Botanical Garden and the local population. To increase our understanding on traditional knowledge and the importance of plant remedies, fieldwork was carried out for 20 days in March 2011 with the aim to identify the most frequent diseases occurring within the Ambalabe community, and to conduct an ethnobotanical survey among the local population. We included four traditional healers to ensure the consistency of information on the use of plants in traditional medicine [16].

\section{Study Site and its surroundings}

The rural community of Ambalabe covers an area of 17437 ha and is located $72 \mathrm{~km}$ northwest of the district capital of Vatomandry, which is the nearest large city and marketplace, in Eastern Madagascar [17]. The community is subject to a humid tropical climate [18], with an average annual rainfall of $1773 \mathrm{~mm}$ and an average annual temperature of $24{ }^{\circ} \mathrm{C}$. Infrastructure decay (disrepair of roads and bridges) led to the isolation of the community and made markets and healthcare options less accessible. The road is only passable in the dry season by $4 \times 4$ vehicles up to $46 \mathrm{~km}$ from Vatomandry. Moreover, the local CSB II cannot meet the demand for medical care of the population given its remoteness from some villages. The rough topography of the area also makes access more difficult. Therefore, people often consult traditional healers instead of doctor.

Ambalabe had 10961 residents in 2013, of which $95 \%$ were farmers (mayor of the rural community of Ambalabe, personal communication). Local inhabitants are mainly Betsimisaraka, for whom shifting cultivation forms the base of their agriculture system [19]. This practice leads to the loss of natural forest [20], including the natural pharmacopeia.

A New Protected Area, Vohibe forest was established in the community in 2008. Vohibe is a humid and evergreen forest of low and medium altitude. It provides to the local population their daily needs such as timber, firewood, medicinal and edible plants. The forest is regularly subjected to the collection of some medicinal plants. It is located in the northwest end of the rural community of Ambalabe, at $48^{\circ} 31^{\prime}$ and $48^{\circ} 36^{\prime} \mathrm{E}$ longitude and $19^{\circ} 06^{\prime}$ and $19^{\circ} 11^{\prime} \mathrm{S}$ latitude, with an altitude ranging from 326 to $1008 \mathrm{~m}$. Vohibe forest is part of Ankeniheny-Zahamena Corridor (CAZ) which is one of the largest remnants of rainforest in the East of Madagascar [21], and it covers an area of 3117 ha (Fig. 1). The forest hosts a wealth of several useful plants, with an endemic species rate of about $70 \%$, nearer to Madagascar's in general [22]. At the end of 2014, near 


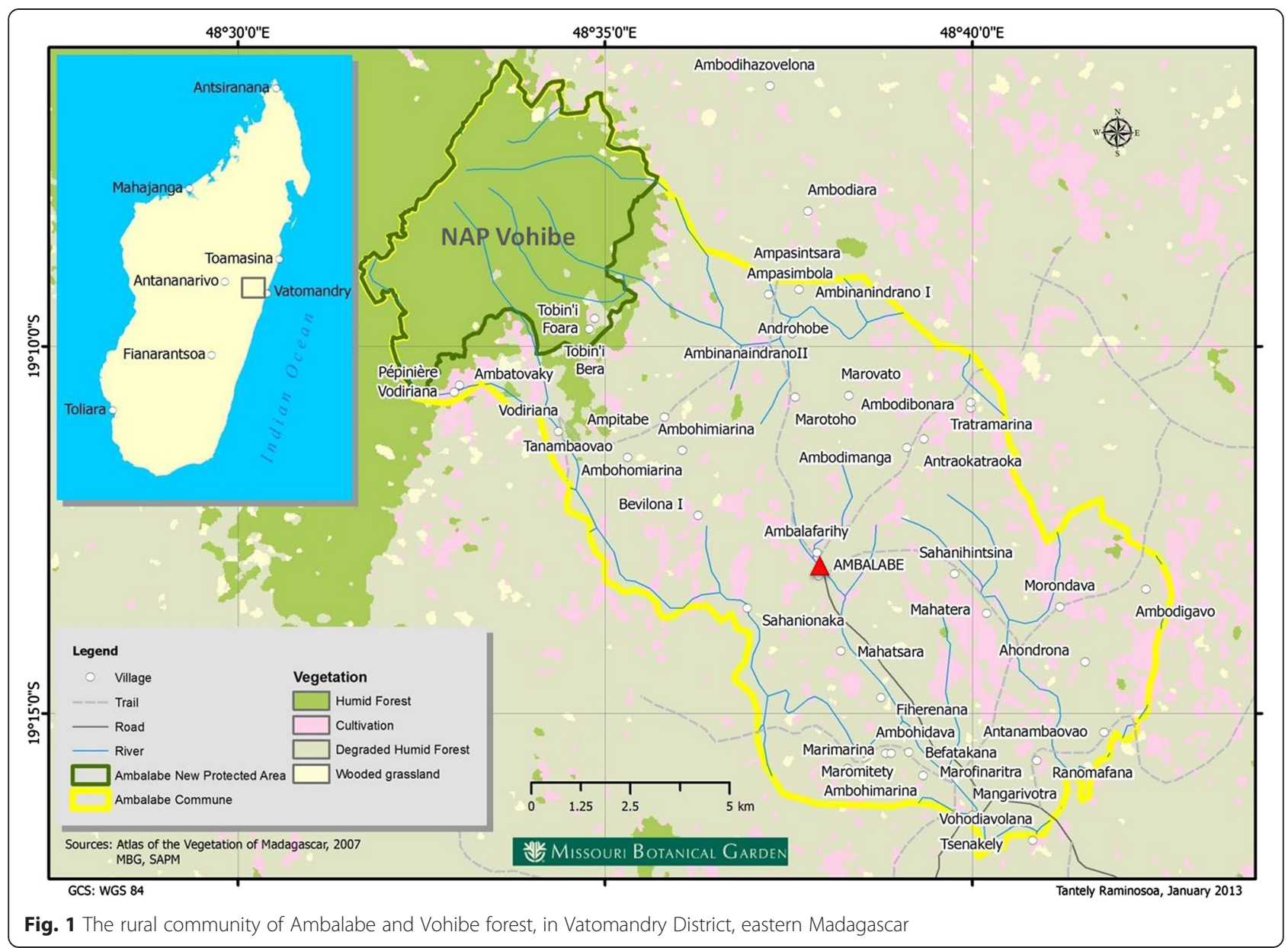

723 species distributed in 113 families and 293 genera were inventoried in Vohibe forest, and near 854 species belonging to 133 families and 355 genera in the whole Ambalabe community, including Vohibe [23].

\section{Ethnobotanical surveys}

Before the surveys, meetings with local authorities, leaders and villagers, were organized to explain the goals of the study and to obtain their prior informed consent [24], based on the Nagoya protocol's rules [25]. All participants were also asked for their prior informed consent before starting interviews. The University ethics commission also approved the study. A collection permit $n^{\circ} 160 / 11 / M E F /$ SG/DGF/DCB.SAP/SCBSE for plants was also presented to the local authorities.

In this study, semi-structured interviews and free listing exercise [26] were conducted among local villages in order to identify the most frequent diseases encountered in the Ambalabe community, and to inventory medicinal plants used by the local population, together with their local names, detailed use information such as parts used and the way to prepare and to administer plant remedies, and also the area of collection. Surveys were also conducted with the local doctor and the four traditional healers. Figure 2 gives the number of informants (apart from the local doctor) according to their occupation. In total, 193 informants from 16 to 86 years old were interviewed, of which $54 \%$ were men and $46 \%$ were women. Most of them are farmers.

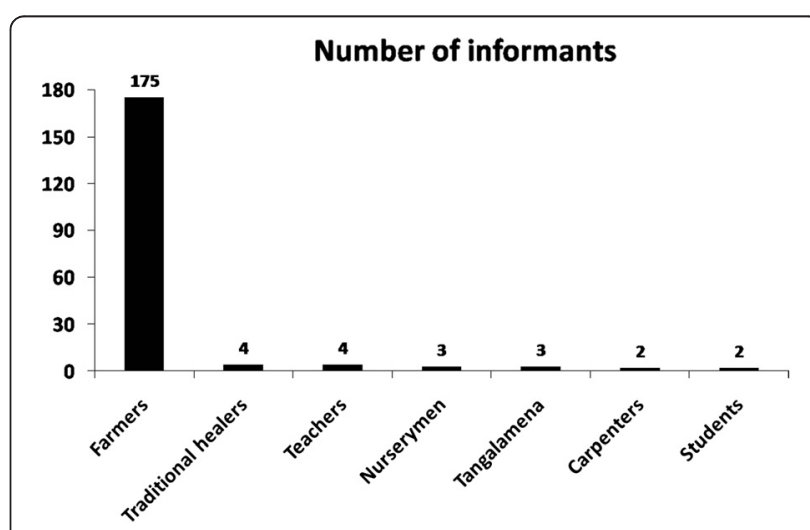

Fig. 2 Number of informants interviewed according to their occupation 
Questionnaires were used as a guide to collect information during the surveys (Additional file 1). Thirteen representative villages of the whole community were visited. The local staff helped us to identify them. Interviews were conducted with both individuals and in group by the first author in Betsimisaraka, the local Malagasy dialect. One local healer acted as a guide and translator if necessary. Plant uses were categorized according to Cámara-Leret et al. [27]. Within the Medicinal and Veterinary category, the following use subcategories were adopted in this study: blood and cardiovascular system; cultural diseases and disorders; dental health; digestive system; endocrine system; general ailments; infections and infestations; metabolic system and nutrition; muscular-skeletal system; nervous system and mental health; poisoning; pregnancy, birth and puerperium; reproductive system and reproductive health; respiratory system; sensory system; skin and subcutaneous tissue; urinary system; veterinary; other.

Local MBG staff has conducted floristic collections in the region since 2004, and has established a reference collection. Given the limited time in the field, common species were directly identified by the local staff in comparison to the reference collection. All species not previously collected for the floristic study were collected and photographed for identification and vouchers were deposited primarily in the national herbarium of the Parc Botanique et Zoologique de Tsimbazaza (TAN). Available duplicates were distributed to the herbaria of Missouri (MO) and the Muséum National d'Histoire Naturelle (P) in Saint Louis and Paris. For common or cosmopolitan plants (for example fruit trees and tropical weeds) found worldwide, vouchers were not collected. For some plant species cited by informants but not encountered during the fieldwork, a brief description of the plant species was taken. Then, the scientific names were elucidated according to vouchers previously collected by researchers in the Ambalabe region or in Madagascar which are available from Tropicos [23] and TAN herbarium.

\section{Statistical analysis}

ANTHROPAC $^{\circ} 4.0$ [28] and XLSTAT ${ }^{\circ}$-Pro 7.5 were used for statistical data analyses. ANTHROPAC, a set of programs using various techniques of collecting "systematic" data [29], was used to analyze the free listing data from which the results were expressed as frequency of citation (\%) and salience (a value that lies between 0 and 1). In this study, frequency is considered as the repetition of citations during the surveys, of which one species related to one specific use of one plant part by one informant is counted as one citation. Salience is a statistic accounting for rank and frequency of species cited [30] in which one species is considered more salient when it appears more often and earlier in freelists.
Species that are frequently cited are assumed to be highly salient, i.e. important to respondents, and species recalled first are assumed to be more salient than species recalled last [31]. Most frequent and most salient species are then considered important for the local population. MannWhitney test at alpha 0.05 , performed through XLSTAT ${ }^{\circ}$ Pro, was used in order to assess the difference between men and women's knowledge, and then simple informants and traditional healers' knowledge on medicinal plants used to treat the most frequent diseases. Kruskal-Wallis test was also used for the age and marital status categories.

\section{Informant consensus}

Another consensus method, which is the fidelity level (FL), was used to quantify the importance of a species for a given disease [32-34]. It calculates a ratio between the number of informants who cited the species for a particular disease $\left(I_{p}\right)$ and the total number of informants that cited the plant for any given disease $\left(I_{u}\right)$. Formula used was:

$$
\mathrm{FL}=\mathrm{I}_{\mathrm{p}} / \mathrm{I}_{\mathrm{u}} \times 100 \%
$$

For the analysis, species with $\mathrm{FL} \geq 75 \%$ were considered as the most relevant for the treatment of a specific disease. However, species only cited once for one ailment, i.e. infrequently cited species, were left out of the analysis.

\section{Results}

In the 13 villages visited, 193 people were interviewed. Of these 89 (46\%) were women and 104 (54\%) were men, ageing from 16 to 86 years. About $49 \%$ of the participants cited frequent diseases encountered in the Ambalabe rural community. Out of 209 species recorded as having medicinal use, belonging to 83 families and 179 genera, 83 species were used to treat the most frequent diseases.

\section{Informants' knowledge}

Our investigations recorded 73 types of illness. The most important of them affect mainly the digestive, the reproductive and the respiratory system. Six of these diseases (diarrhea, malaria, stomach-ache, cough, bilharzia and dysentery) were identified as the most frequent ailments in the Ambalabe community. Local people used 83 different plant species belonging to 49 families and 77 genera to treat these six afflictions, i.e. an average of 17 species for each of them. Seventy-seven species were identified to species level and $29 \%$ were endemic. About $23 \%$ of the 83 species are known by at least ten informants. Sixteen species were used to treat more than one ailment. The number of species used for each disease is shown in Table 1. Most of the species were used to treat 
Table 1 Number of species which treat the six frequent diseases in the Ambalabe rural community

\begin{tabular}{ll}
\hline Diseases & Number of species used \\
\hline Bilharzia & 6 \\
Cough & 14 \\
Diarrhea & 32 \\
Dysentery & 6 \\
Malaria & 13 \\
Stomach-ache & 30 \\
\hline
\end{tabular}

diarrhea and stomach-ache. Fewer medicinal plants were used for bilharzia and dysentery treatment. People often consulted a doctor for these two serious ailments. Table 2 gives the informants' knowledge according to demographic variables. Men cited more plant species as used than women. This might be a residual effect of the higher number of male informants interviewed. However, when analyzing the average number of species cited by each informant in relation to gender, a Mann-Whitney test showed that men held more knowledge than women, with $P=0.01<0.05$. This difference is significant. Men were also the only informant group who cited all six species used to treat bilharzia. Within the age and marital status categories, the difference on plant species cited is not significant with respectively $P=0.6$ and $P=0.9$. However, it should be noted that the single widowed informant had an important knowledge by citing nine species, nearly two species for each of the four ailments he cited.

When comparing traditional healers and simple informants' knowledge on plant species used to treat the most frequent diseases, a Mann-Whitney test showed that no significant difference was found concerning their knowledge $(P=0.8>0.05)$. This means that both informant groups cite almost the same amount of plants (an average of two species per ailment) used to treat each disease. However, cited plant species were different according to the informant, which explains the high number of plants recorded (83 species) for the six ailments.

Therefore, difference was only found among the gender setting. No difference was found between traditional healers and simple informants' knowledge, which means that the more these diseases are frequent, the more people get to know plant species used to treat them. As such, the local population did often not consult traditional healers or the local doctor except for treating bilharzia and dysentery for which few plants are known as effective, and which are considered as diseases with high risk of complications.

\section{Frequent diseases and medicinal plants used}

A free listing analysis highlighted the 11 plant species most commonly used for the treatment of five of the six frequent diseases, with a frequency higher than $5 \%$ (Table 3). Three of them (Kalanchoe prolifera, Paederia thouarsiana, Catharanthus roseus) are endemic to Madagascar, six (Mollugo nudicaulis, Litchi chinensis, Rubus moluccanus, Petchia erythrocarpa, Harungana madagascariensis, Aeschynomene sensitiva) are not endemic and two (Psidium guajava, Clidemia hirta) are naturalized. The most important were Mollugo nudicaulis, Litchi chinensis, Kalanchoe prolifera and Paederia thouarsiana with more than $10 \%$ of frequency. Mollugo nudicaulis was the most frequent as well as the most salient species used, thus assumed to be important for the local population. Leaves were the most important plant part used for treatment. Remedies were basically prepared as decoction, which was administered orally.

None of the top eleven species was used for bilharzia treatment. However, six different species were specifically

Table 2 Informants' knowledge in the Ambalabe rural community according to demographic variables

\begin{tabular}{|c|c|c|c|c|c|c|}
\hline & & $\begin{array}{l}\text { Total number of people } \\
\text { interviewed }\end{array}$ & $\begin{array}{l}\text { Number of informants who } \\
\text { cited frequent diseases }\end{array}$ & $\begin{array}{l}\text { Number of diseases cited } \\
\text { (not cited) }\end{array}$ & $\begin{array}{l}\text { Total of species } \\
\text { cited }\end{array}$ & $\begin{array}{l}\text { Percentage of } \\
\text { total }\end{array}$ \\
\hline \multirow[t]{2}{*}{ Gender } & Men & 104 & 58 & 6 & 68 & 82 \\
\hline & Women & 89 & 36 & 5 (bilharzia) & 45 & 54.2 \\
\hline \multirow[t]{6}{*}{ Age group } & {$[16-25]$} & 43 & 15 & 6 & 22 & 26.5 \\
\hline & {$[26-35]$} & 38 & 20 & 6 & 28 & 33.7 \\
\hline & {$[36-45]$} & 44 & 25 & 6 & 37 & 44.6 \\
\hline & {$[46-55]$} & 34 & 21 & 6 & 40 & 48.2 \\
\hline & {$[56-65]$} & 20 & 9 & 6 & 26 & 31.3 \\
\hline & {$[66+]$} & 14 & 4 & 4 (bilharzia, dysentery) & 4 & 4.8 \\
\hline \multirow[t]{4}{*}{ Marital status } & Single & 30 & 13 & 4 (bilharzia, dysentery) & 16 & 19.3 \\
\hline & Married & 152 & 76 & 6 & 78 & 94 \\
\hline & Divorcee & 7 & 4 & 4 (bilharzia, stomach-ache) & 7 & 8.4 \\
\hline & Widowed & 4 & 1 & 4 (cough, malaria) & 9 & 10.8 \\
\hline
\end{tabular}


Table 3 Eleven most common species used to treat frequent diseases in the Ambalabe rural community

\begin{tabular}{|c|c|c|c|c|c|c|c|c|c|}
\hline Family & Scientific name & $\begin{array}{l}\text { Local name } \\
\text { (dialect: Betsimisaraka) }\end{array}$ & $\begin{array}{l}\text { Diseases } \\
\text { treated }\end{array}$ & $\begin{array}{l}\text { Parts } \\
\text { used }\end{array}$ & $\begin{array}{l}\text { Preparation } \\
\text { method }\end{array}$ & Administration & $\begin{array}{l}\text { Frequency } \\
(\%)\end{array}$ & Salience & $\begin{array}{l}\text { Voucher } \\
\text { number }\end{array}$ \\
\hline Molluginaceae & $\begin{array}{l}\text { Mollugo nudicaulis } \\
\text { Lam. }\end{array}$ & Aferotany & $\begin{array}{l}\text { Malaria, } \\
\text { stomach-ache }\end{array}$ & $\begin{array}{l}\text { Whole } \\
\text { plant }\end{array}$ & $\begin{array}{l}\text { Decoction, } \\
\text { infusion }\end{array}$ & Oral & 21.3 & 0.15 & RKN 485 \\
\hline Sapindaceae & $\begin{array}{l}\text { Litchi chinensis } \\
\text { Sonn. }\end{array}$ & Letisia & $\begin{array}{l}\text { Diarrhea, } \\
\text { dysentery, } \\
\text { stomach-ache }\end{array}$ & $\begin{array}{l}\text { Bark, } \\
\text { Leaves }\end{array}$ & Decoction & Oral & 12.8 & 0.1 & \\
\hline Crassulaceae & $\begin{array}{l}\text { Kalanchoe prolifera } \\
\text { (Bowie ex Hook.) } \\
\text { Raym.-Hamet }\end{array}$ & Sodifafana & $\begin{array}{l}\text { Cough, } \\
\text { malaria }\end{array}$ & Leaves & $\begin{array}{l}\text { Decoction, } \\
\text { heat and } \\
\text { press the } \\
\text { juice }\end{array}$ & Oral & 11.7 & 0.08 & RKN 512 \\
\hline Rubiaceae & $\begin{array}{l}\text { Paederia thouarsiana } \\
\text { Baill. }\end{array}$ & $\begin{array}{l}\text { Vahivola, } \\
\text { vahimantsina }\end{array}$ & Stomach-ache & $\begin{array}{l}\text { Branch, } \\
\text { leaves }\end{array}$ & Decoction & Oral & 10.6 & 0.08 & RA 1349 \\
\hline Apocynaceae & $\begin{array}{l}\text { Catharanthus roseus } \\
\text { (L.) G. Don }\end{array}$ & Arivotaombelona & Malaria & Leaves & Decoction & Oral & 7.4 & 0.03 & $\begin{array}{l}\text { RKN 503, } \\
504\end{array}$ \\
\hline Rosaceae & Rubus moluccanus $\mathrm{L}$. & Takoaka & $\begin{array}{l}\text { Diarrhea, } \\
\text { dysentery }\end{array}$ & Leaves & $\begin{array}{l}\text { Crush, } \\
\text { decoction }\end{array}$ & Oral & 7.4 & 0.07 & REH 720 \\
\hline Myrtaceae & Psidium guajava L. & $\begin{array}{l}\text { Gavo, gavombazaha, } \\
\text { gavobe }\end{array}$ & $\begin{array}{l}\text { Diarrhea, } \\
\text { dysentery }\end{array}$ & $\begin{array}{l}\text { Bark, } \\
\text { leaves }\end{array}$ & Decoction & Oral & 7.4 & 0.06 & RCS 456 \\
\hline Melastomataceae & $\begin{array}{l}\text { Clidemia hirta (L.) } \\
\text { D. Don }\end{array}$ & Sompatra & $\begin{array}{l}\text { Diarrhea, } \\
\text { malaria, } \\
\text { stomach-ache }\end{array}$ & $\begin{array}{l}\text { Leaves, } \\
\text { roots }\end{array}$ & Decoction & $\begin{array}{l}\text { Inhalation, } \\
\text { oral }\end{array}$ & 6.4 & 0.06 & RKN 513 \\
\hline Apocynaceae & $\begin{array}{l}\text { Petchia erythrocarpa } \\
\text { (Vatke) Leeuwenb. }\end{array}$ & Hintona & Malaria & $\begin{array}{l}\text { Bark, } \\
\text { leaves }\end{array}$ & $\begin{array}{l}\text { Decoction, } \\
\text { infusion }\end{array}$ & Oral & 6.4 & 0.05 & RKN 453 \\
\hline Hypericaceae & $\begin{array}{l}\text { Harungana } \\
\text { madagascariensis } \\
\text { Lam. ex Poir. }\end{array}$ & Harongana & Diarrhea & $\begin{array}{l}\text { Bark, } \\
\text { leaves }\end{array}$ & Decoction & Oral & 6.4 & 0.03 & RA 1325 \\
\hline Fabaceae & $\begin{array}{l}\text { Aeschynomene } \\
\text { sensitiva Sw. }\end{array}$ & Fanombo tintina & Malaria & Leaves & Decoction & Oral & 5.3 & 0.05 & RKN 523 \\
\hline
\end{tabular}

Table 4 Relevant species with high fidelity level used per disease category

\begin{tabular}{|c|c|c|c|c|}
\hline Disease & Relevant species & Distribution & Number of citations & $\mathrm{FL}$ \\
\hline Bilharzia & Senna alata (L.) Roxb. & Naturalized & 2 & 100 \\
\hline \multirow[t]{2}{*}{ Cough } & Citrus limon (L.) Burm. f. & Naturalized & 3 & 100 \\
\hline & Oxalis corniculata $\mathrm{L}$. & Naturalized & 3 & 100 \\
\hline \multirow[t]{9}{*}{ Diarrhea } & Artocarpus heterophyllus Lam. & Not endemic & 4 & 100 \\
\hline & Canarium L. & Endemic & 4 & 100 \\
\hline & Raphia farinifera (Gaertn.) Hyl. & Naturalized & 4 & 100 \\
\hline & Danais terminalis Boivin ex Drake & Endemic & 3 & 100 \\
\hline & Macaranga obovata Boivin ex Baill. & Endemic & 3 & 100 \\
\hline & Musa paradisiaca L. & Not endemic & 3 & 100 \\
\hline & Psidium cattleyanum Sabine & Naturalized & 3 & 100 \\
\hline & Maesa lanceolata Forssk. & Naturalized & 2 & 100 \\
\hline & Manihot esculenta Crantz & Not endemic & 4 & 80 \\
\hline \multirow[t]{2}{*}{ Malaria } & Catharanthus roseus (L.) G. Don & Endemic & 7 & 100 \\
\hline & Aeschynomene sensitiva Sw. & Not endemic & 5 & 83 \\
\hline \multirow[t]{2}{*}{ Stomach-ache } & Cyanthillium cinereum (L.) H. Rob. & Not endemic & 2 & 100 \\
\hline & Paederia thouarsiana Baill. & Endemic & 10 & 77 \\
\hline
\end{tabular}


Table 5 Medicinal plants used to treat six most frequent diseases in Ambalabe rural community, Madagascar

\begin{tabular}{|c|c|c|c|c|c|c|c|c|c|}
\hline Family & Scientific name & Local name & $\begin{array}{l}\text { Diseases } \\
\text { treated }\end{array}$ & Part used & Preparation & Administration & $\begin{array}{l}\text { Number of } \\
\text { citations }\end{array}$ & $\mathrm{FL}$ & Voucher \\
\hline \multirow[t]{2}{*}{ Anacardiaceae } & \multirow[t]{2}{*}{ Sorindeia madagascariensis DC. } & \multirow[t]{2}{*}{ Voasirindrina } & Diarrhea & Leaves & Decoction & Oral & 3 & 27 & RA 1334 \\
\hline & & & Stomach-ache & Leaves & Decoction & Oral & 1 & 9 & \\
\hline Annonaceae & Annona muricata L. & Voatsokina, goronoa & Stomach-ache & Leaves & Decoction & Oral & 1 & 50 & CR 4242 \\
\hline Aphloiaceae & Aphloia theiformis (Vahl) Benn. & Fandramanana & Stomach-ache & Leaves & Decoction & Oral & 1 & 17 & RA 1335 \\
\hline Apiaceae & Centella asiatica (L.) Urb. & Talapetraka & Stomach-ache & Leaves & Decoction & Oral & 1 & 100 & RNH 545 \\
\hline \multirow[t]{3}{*}{ Apocynaceae } & Catharanthus roseus (L.) G. Don & Arivotaombelona & Malaria & Leaves & Decoction & Oral & 7 & 100 & RKN 503, 504 \\
\hline & \multirow[t]{2}{*}{ Petchia erythrocarpa (Vatke) Leeuwenb. } & \multirow[t]{2}{*}{ Hintona } & \multirow[t]{2}{*}{ Malaria } & Leaves & Decoction & Oral & 6 & 33 & RKN 453 \\
\hline & & & & Bark & Infusion & Oral & & & \\
\hline \multirow[t]{3}{*}{ Arecaceae } & \multirow[t]{2}{*}{ Cocos nucifera $\mathrm{L}$. } & \multirow[t]{2}{*}{ Coco } & Diarrhea & Leaves & Infusion & Oral & 1 & 50 & Gunn 643 \\
\hline & & & Stomach-ache & Leaves & Decoction & Oral & 1 & 50 & \\
\hline & Raphia farinifera (Gaertn.) Hyl. & Rafia & Diarrhea & Fruit & Decoction & Oral & 4 & 100 & \\
\hline \multirow[t]{8}{*}{ Asteraceae } & Cyanthillium cinereum (L.) H. Rob. & Ramitsiry & Stomach-ache & Whole plant & Decoction & Oral & 2 & 100 & AP 4968 \\
\hline & \multirow[t]{2}{*}{ Elephantopus scaber L. } & \multirow[t]{2}{*}{ Angadoha } & Diarrhea & Leaves & Crush and heat & Oral & 1 & 14 & \\
\hline & & & Stomach-ache & Leaves & Heat and press & Oral & 2 & 29 & \\
\hline & \multirow[t]{2}{*}{ Emilia citrina DC. } & \multirow[t]{2}{*}{ Tsihontsihona } & Malaria & Whole plant & Decoction & Oral & 2 & 22 & RKN 448 \\
\hline & & & Stomach-ache & Leaves & Decoction & Oral & 2 & 22 & \\
\hline & Helianthus annuus L. & Tanatanamasoandro & Malaria & Leaves & $\begin{array}{l}\text { Infusion, } \\
\text { decoction }\end{array}$ & Oral & 3 & 38 & \\
\hline & Helichrysum flagellare Baker & Ahidroranga & Stomach-ache & Leaves & Decoction & Oral & 2 & 11 & RKN 548 \\
\hline & Psiadia altissima (DC.) Drake & Dingadingana & Diarrhea & Leaves & Decoction & Oral & 1 & 8 & FRB 194 \\
\hline Burseraceae & Canarium L. & Ramy & Diarrhea & Bark & Decoction & Oral & 4 & 100 & RZA 1186 \\
\hline \multirow[t]{2}{*}{ Clusiaceae } & $\begin{array}{l}\text { Garcinia chapelieri (Planch. \& Triana) } \\
\text { H. Perrier }\end{array}$ & Takasina & Cough & Leaves & Decoction & Oral & 1 & 100 & RKN 473 \\
\hline & $\begin{array}{l}\text { Symphonia fasciculata (Noronha ex } \\
\text { Thouars) Vesque }\end{array}$ & Kijy & Diarrhea & Bark & Decoction & Oral & 1 & 100 & RAB 66 \\
\hline Combretaceae & Combretum Loefl. & Vahinaletra & Stomach-ache & Leaves & Decoction & Oral & 1 & 100 & RA 1323 \\
\hline Connaraceae & Cnestis polyphylla Lam. & Sefana & Diarrhea & Stem & Decoction & Oral & 1 & 100 & RKN 511 \\
\hline \multirow[t]{2}{*}{ Crassulaceae } & $\begin{array}{l}\text { Kalanchoe prolifera (Bowie ex Hook.) Raym.- } \\
\text { Hamet }\end{array}$ & Sodifafana & Malaria & Leaves & Decoction & Oral & 4 & 31 & RKN 512 \\
\hline & & & Cough & Leaves & Heat and press & Oral & 7 & 54 & \\
\hline \multirow[t]{2}{*}{ Cucurbitaceae } & \multirow[t]{2}{*}{ Momordica charantia L. } & \multirow[t]{2}{*}{ Margôzy } & Malaria & Leaves & Decoction & Oral & 2 & 67 & RZK 3096 \\
\hline & & & Stomach-ache & Leaves & Decoction & Oral & 2 & 67 & \\
\hline Cucurbitaceae & Unidentified & Voatangolehy & Bilharzia & Leaves & Heat and press & Oral & 1 & 100 & \\
\hline
\end{tabular}


Table 5 Medicinal plants used to treat six most frequent diseases in Ambalabe rural community, Madagascar (Continued)

\begin{tabular}{|c|c|c|c|c|c|c|c|c|c|}
\hline Cunoniaceae & Weinmannia bojeriana Tul. & Sokia & Dysentery & Bark & Decoction & Oral & 1 & 100 & RZA 533 \\
\hline \multirow[t]{2}{*}{ Euphorbiaceae } & Macaranga obovata Boivin ex Baill. & Mankaranana & Diarrhea & Bark & Decoction & Oral & 3 & 100 & RA 1051 \\
\hline & Manihot esculenta Crantz & Mangahazo & Diarrhea & Leaves & Decoction & Oral & 4 & 80 & \\
\hline \multirow[t]{6}{*}{ Fabaceae } & Aeschynomene sensitiva Sw. & Fanombo tintina & Malaria & Leaves & Decoction & Oral & 5 & 83 & RKN 523 \\
\hline & Dalbergia monticola Bosser \& R. Rabev. & Hitsika & Bilharzia & Wood-heart & Decoction & Oral & 1 & 100 & Perrier 4830 \\
\hline & $\begin{array}{l}\text { Desmodium } \\
\text { ramosissimum G. Don }\end{array}$ & Tsilavondrivotra & Diarrhea & Leaves & Heat and & Oral & 3 & 60 & RKN 516 \\
\hline & & & Cough & Leaves & Decoction & Oral & 1 & 20 & \\
\hline & Entada gigas (L.) Fawc. \& Rendle & Vahinkarabo & Diarrhea & Leaves, stem & Decoction & Oral & 2 & 13 & MAR 13 \\
\hline & Senna alata (L.) Roxb. & 4 épingles & Bilharzia & Leaves & Decoction & Oral & 2 & 100 & RKN 490 \\
\hline \multirow[t]{2}{*}{ Gentianaceae } & Exacum quinquenervium Griseb. & Mamoahely & Diarrhea & Leaves & Decoction & Oral & 1 & 100 & ROR 842 \\
\hline & Ornichia madagascariensis (Baker) Klack. & Aferotaniala & Malaria & Whole plant & Decoction & Oral & 1 & 100 & RKN 496 \\
\hline Gleicheniaceae & Sticherus flagellaris (Bory ex Willd.) Ching & Rangontohitra & Diarrhea & Leaves & Decoction & Oral & 1 & 100 & RZK 6632 \\
\hline Hypericaceae & $\begin{array}{l}\text { Harungana madagascariensis Lam. } \\
\text { ex Poir. }\end{array}$ & Harongana & Diarrhea & Bark, leaves & Decoction & Oral & 6 & 27 & RA 1325 \\
\hline Lamiaceae & Plectranthus perrieri Hedge & Amparimaso & Diarrhea & Leaves & Heat and press & Oral & 1 & 100 & $\begin{array}{l}\text { Descoings } \\
3703\end{array}$ \\
\hline Lygodiaceae & Lygodium lanceolatum Desv. & Famalotrakanga & Stomach-ache & Leaves & Decoction & Oral & 1 & 17 & RKN 446 \\
\hline \multirow[t]{4}{*}{ Melastomataceae } & Clidemia hirta (L.) D. Don & Sompatra & Diarrhea & Leaves & Decoction & Oral & 4 & 22 & RKN 513 \\
\hline & & & Malaria & Leaves & Decoction & $\begin{array}{l}\text { Inhalation, } \\
\text { oral }\end{array}$ & 1 & 6 & \\
\hline & & & Stomach-ache & Roots & Decoction & Oral & 1 & 6 & \\
\hline & Dichaetanthera oblongifolia Baker & Tsitrotroka & Stomach-ache & Leaves & Decoction & Oral & 1 & 100 & RA 1339 \\
\hline Meliaceae & Melia azedarach L. & Voandelaka & Malaria & Leaves & Decoction & Oral & 2 & 11 & RKN 447 \\
\hline \multirow[t]{2}{*}{ Molluginaceae } & Mollugo nudicaulis Lam. & Aferotany & Malaria & Whole plant & Decoction & Oral & 19 & 66 & RKN 485 \\
\hline & & & Stomach-ache & Whole plant & Infusion & Oral & 1 & 3 & \\
\hline \multirow[t]{4}{*}{ Moraceae } & Artocarpus heterophyllus Lam. & Ampalibe & Diarrhea & Leaves & Crush & Oral & 4 & 100 & LRZ 1838 \\
\hline & Ficus polita Vahl & Mandresy & Stomach-ache & Leaves & Decoction & Oral & 1 & 7 & RKN 449 \\
\hline & Ficus reflexa Thunb. & Nonoka madinika & Cough & Leaves & Decoction & Oral & 1 & 25 & RKN 455 \\
\hline & Streblus dimepate (Bureau) C.C. Berg & Manasavelona & Diarrhea & Leaves & Decoction & Oral & 1 & 17 & RKN 552 \\
\hline \multirow[t]{4}{*}{ Musaceae } & Musa paradisiaca $\mathrm{L}$. & Akondro & Diarrhea & Fruit & Paste & Oral & 3 & 100 & \\
\hline & & & & Inflorescence & Decoction & Oral & & & \\
\hline & & & & Resin & & Oral & & & \\
\hline & & & Dysentery & Inflorescence & Heat and press & Oral & 1 & 33 & \\
\hline Myristicaceae & & Ilon-draharaha & Cough & Seeds & Oil & Topical & 1 & 20 & RA 972 \\
\hline
\end{tabular}


Table 5 Medicinal plants used to treat six most frequent diseases in Ambalabe rural community, Madagascar (Continued)

\begin{tabular}{|c|c|c|c|c|c|c|c|c|c|}
\hline & $\begin{array}{l}\text { Mauloutchia humblotii (H. Perrier) } \\
\text { Capuron }\end{array}$ & & & & & & & & \\
\hline \multirow[t]{6}{*}{ Myrtaceae } & Eucalyptus camaldulensis Dehnh. & Kininina & Malaria & Young leaves & Decoction & Oral & 1 & 50 & \\
\hline & & & Diarrhea & Leaves & Decoction & Oral & 1 & 50 & \\
\hline & Psidium cattleyanum Sabine & Gavo tsinahy & Diarrhea & Leaves & Decoction & Oral & 3 & 100 & Gentry 11251 \\
\hline & Psidium guajava $\mathrm{L}$. & $\begin{array}{l}\text { Gavo, gavombazaha, } \\
\text { gavobe }\end{array}$ & Diarrhea & Leaves & Decoction & Oral & 6 & 35 & RCS 456 \\
\hline & & & Dysentery & Bark & Decoction & Oral & 1 & 6 & \\
\hline & $\begin{array}{l}\text { Syzygium malaccense (L.) Merr. \& } \\
\text { L.M. Perry }\end{array}$ & Makoba & Diarrhea & Roots & Decoction & Oral & 1 & 100 & D'Arcy 15233 \\
\hline Orchidaceae & Aerangis hyaloides (Rchb. f.) Schltr. & Tsiakondroakondro & Cough & Leaves & Heat and press & Oral & 1 & 100 & AP 7155 \\
\hline \multirow[t]{2}{*}{ Oxalidaceae } & Oxalis corniculata $\mathrm{L}$. & Takasintany & Cough & Whole plant & Decoction & Oral & 3 & 100 & AP 5034 \\
\hline & & & & Whole plant & Heat and press & Oral & & & \\
\hline Pandanaceae & Pandanus sp. Parkinson & Manasa ala & Cough & Leaves & Decoction & Oral & 1 & 100 & \\
\hline Passifloraceae & Passiflora edulis Sims & Garana madinika & Diarrhea & Leaves & Crush and press & Oral & 2 & 50 & RKN 456 \\
\hline Phyllanthaceae & Phyllanthus nummulariifolius Poir. & Mandrihariva & Stomach-ache & Leaves & Decoction & Oral & 1 & 33 & RKN 542 \\
\hline Piperaceae & Piper borbonense (Miq.) C. DC. & $\begin{array}{l}\text { Tsimahalatsaka, } \\
\text { voantsiperifery }\end{array}$ & Stomach-ache & Leaves & Decoction & Oral & 1 & 33 & RA 941 \\
\hline Pittosporaceae & Pittosporum ochrosiifolium Bojer & Hazombary, maimbovitsika & Cough & Leaves & Decoction & Oral & 2 & 50 & RA 1322 \\
\hline \multirow[t]{2}{*}{ Poaceae } & Oryza sativa $\mathrm{L}$. & Vary & Dysentery & Seeds & Cook and filter & Oral & 1 & 17 & \\
\hline & Zea mays $\mathrm{L}$. & Tsakotsako & Stomach-ache & Stem & Decoction & Oral & 1 & 100 & \\
\hline Primulaceae & Maesa lanceolata Forssk. & Radoka & Diarrhea & Leaves & Decoction & Oral & 2 & 100 & RKN 500 \\
\hline Pteridaceae & Pteris cf. cretica L. & Ravimbolo & Stomach-ache & Leaves & Decoction & Oral & 1 & 5 & RKN 458 \\
\hline Pteridophyta & Unidentified & Ahitrimpa & Cough & Leaves & Decoction & Oral & 1 & 100 & \\
\hline Rhamnaceae & Gouania tiliifolia Lam. & Ranovavanaomby & Cough & Leaves & Crush & Oral & 1 & 6 & RKN 499 \\
\hline \multirow[t]{4}{*}{ Rosaceae } & Eriobotrya japonica (Thunb.) Lindl. & Pibasy & Cough & Leaves & Decoction & Oral & 2 & 50 & Croat 32156 \\
\hline & Rubus moluccanus L. & Takoaka & Diarrhea & Leaves & Crush, decoction & Oral & 6 & 60 & REH 720 \\
\hline & & & Dysentery & Leaves & Decoction & Oral & 1 & 10 & \\
\hline & Rubus rosifolius Sm. & Voandroy & Stomach-ache & Leaves & Decoction & Oral & 1 & 33 & PPL 6592 \\
\hline \multirow[t]{3}{*}{ Rubiaceae } & Breonia decaryana Homolle & Molompangady & Bilharzia & $\begin{array}{l}\text { Bark and } \\
\text { leaves }\end{array}$ & Decoction & Oral & 1 & 20 & RZA 158 \\
\hline & Danais terminalis Boivin ex Drake & Vahinofokorana & Diarrhea & Roots & Decoction & Oral & 3 & 100 & RKN 680 \\
\hline & Paederia thouarsiana Baill. & Vahivola, vahimantsina & Stomach-ache & $\begin{array}{l}\text { Branch, } \\
\text { leaves }\end{array}$ & Decoction & Oral & 10 & 77 & RA 1349 \\
\hline Rutaceae & Citrus aurantium L. & Voahangy ala & Stomach-ache & Young leaves & Decoction & Oral & 1 & 33 & AP 5569 \\
\hline
\end{tabular}


Table 5 Medicinal plants used to treat six most frequent diseases in Ambalabe rural community, Madagascar (Continued)

\begin{tabular}{|c|c|c|c|c|c|c|c|c|c|}
\hline & Citrus limon (L.) Burm. f. & Voahangitsoha & Cough & Fruit & Juice & Oral & 3 & 100 & \\
\hline & & & & Leaves & Decoction & Oral & & & \\
\hline & Citrus reticulata Blanco & Mandarinina & Bilharzia & Leaves & Decoction & Oral & 1 & 50 & \\
\hline & Toddalia asiatica (L.) Lam. & Anakasimba & Malaria & Leaves & Decoction & Oral & 1 & 50 & RA 1329 \\
\hline & & & Stomach-ache & Leaves & Decoction & Oral & 1 & 50 & \\
\hline \multirow[t]{3}{*}{ Sapindaceae } & Litchi chinensis Sonn. & Letisia & Diarrhea & Leaves & Decoction & Oral & 8 & 67 & \\
\hline & & & Dysentery & Bark & Decoction & Oral & 2 & 17 & \\
\hline & & & Stomach-ache & Leaves & Decoction & Oral & 2 & 17 & \\
\hline Sarcolaenaceae & Schizolaena Thouars & Kikazana & Stomach-ache & Leaves & Decoction & Oral & 2 & 67 & \\
\hline \multirow[t]{3}{*}{ Solanaceae } & Capsicum annuum L. & Pilopilo & Stomach-ache & Fruit & Crush & Oral & 1 & 33 & ALJ 1183 \\
\hline & Lycopersicon esculentum Mill. & Voatabia & Diarrhea & Leaves & Heat and press & Oral & 1 & 100 & \\
\hline & Solanum mauritianum Scop. & Bakobako & Diarrhea & Leaves & Crush and press & Oral & 2 & 40 & $\begin{array}{l}\text { Schlieben } \\
8097\end{array}$ \\
\hline Strelitziaceae & Ravenala madagascariensis Sonn. & Fontsy & Stomach-ache & Young leaves & Decoction & Oral & 2 & 67 & CR 5205 \\
\hline \multirow[t]{2}{*}{ Verbenaceae } & Lantana camara L. & Radriaka & Diarrhea & Leaves & Decoction & Oral & 1 & 7 & GES 1601 \\
\hline & & & Stomach-ache & Leaves & Decoction & Oral & 3 & 21 & \\
\hline \multirow[t]{3}{*}{ Zingiberaceae } & Aframomum angustifolium (Sonn.) K. Schum. & Lingoza & Cough & Fruit & Decoction & Oral & 1 & 25 & GES 1624 \\
\hline & Curcuma longa L. & Tamotamo & Stomach-ache & Tuber & Decoction & Oral & 2 & 40 & Geay 8277 \\
\hline & Zingiber zerumbet (L.) Roscoe ex Sm. & Sakarivondambo & Bilharzia & Tuber & Decoction & Oral & 4 & 40 & RKN 443 \\
\hline
\end{tabular}


used to treat this disease (Breonia decaryana, Citrus reticulata, Dalbergia monticola, Senna alata, Zingiber zerumbet and one Cucurbitaceae). Participants did however show a limited knowledge of plants to treat bilharzia.

Concerning the locations of harvest, our study found that only $38.6 \%$ of the 83 recorded medicinal plants occurred in Vohibe forest. Most species were collected outside the protected area. Of these $19.3 \%$ were cultivated and the remaining were collected in the surroundings of the villages, in house yards, or in some crop fields. Although many of these species might be considered common, some occur only in small forest fragments, and might thus easily be threatened.

\section{Fidelity level}

Most relevant species for each disease, according to their fidelity, are given in Table 4 with their number of citations. About $31 \%$ of them were endemic to Madagascar. One species was relevant for bilharzia, two species for cough, nine species for diarrhea (of which three were endemic) and also two species each for malaria and stomach-ache (one species for each was also endemic). No species was identified as relevant for the dysentery category, because people normally consulted the local doctor for this ailment. The number of citations for the 16 relevant species ranged from two to ten. Only Paederia thouarsiana has ten numbers of citations. It is annotated that plant species frequently cited are not always the most relevant for the treatment of one disease. The Table 5 gives more details on the 83 species inventoried as medicinal plants used for the six frequent ailments encountered in the Ambalabe community, with their uses and their fidelity level.

\section{Discussion}

The use of herbal medicine often reflects a lack of access to modern medicine. Our study focused on medicinal plants used to treat the most frequent diseases encountered in the rural community of Ambalabe and their degree of threats.

The six diseases identified are most common in rural areas in Madagascar, especially those which affect the digestive system $[7,8,35]$, and some of them are sometimes considered as major threats in tropical and subtropical countries $[36,37]$. However, plant species used are generally diverse, even in the same study area. As well, uses are sometimes different for each plant species cited. Yet, it is very common for one species to be used to treat more than one disease. Informants play an important role on this traditional knowledge richness. This indicates how important the role of an ethnobotanical investigation is on documenting and archiving this cultural inheritance.

Rabearivony et al. [7] conducted a similar study in Ambalabe by documenting the medicinal plants known by men. By considering only the medicinal plants used for the six frequent diseases, the results highlight some similarity and also clear differences between the two studies (Table 6). Species used for diarrhea and stomachache treatment were always abundant in the two studies. Yet, no plant species were recorded for dysentery in Rabearivony et al. Concerning the total number of species inventoried, our study found more species used for each disease (except for bilharzia and malaria which are more similar), and only 20 species were common. When compared to other studies conducted in some areas in Madagascar, the number of common species decreased and some literature sources did not give a list of species used for one or two ailments (often bilharzia and dysentery), indicating that each region/locality has its own set of medicinal plants used. Such results highlight the importance of traditional medicine and the diversity of plant species used in the lives of Malagasy people. In this study, the high number of species used reflects the botanical richness of Ambalabe and also the considerable traditional knowledge of the local population, which deserves to be preserved.

Table 6 Comparison of the present study to other studies conducted in Ambalabe and in Madagascar: species considered are those used for the six frequent diseases

\begin{tabular}{|c|c|c|c|c|c|c|c|}
\hline & & $\begin{array}{l}\text { Present } \\
\text { study }\end{array}$ & $\begin{array}{l}\text { Rabearivony } \\
\text { et al. [7] }\end{array}$ & Rakotonandrasana [39] & Razafindraibe [8] & Quansah [19] & Nicolas [38] \\
\hline Total number of species & & 83 & 62 & 22 & 65 & 7 & 81 \\
\hline Common species used & & & 20 & 2 & 12 & 4 & 9 \\
\hline \multirow[t]{6}{*}{ Number of species per disease } & Bilharzia & 6 & 7 & 0 & 0 & 0 & 1 \\
\hline & Cough & 14 & 12 & 9 & 18 & 0 & 20 \\
\hline & Diarrhea & 32 & 20 & 6 & 21 & 2 & 41 \\
\hline & Dysentery & 6 & 0 & 0 & 6 & 3 & 28 \\
\hline & Malaria & 13 & 14 & 5 & 25 & 0 & 17 \\
\hline & Stomach-ache & 30 & 25 & 4 & 12 & 3 & 10 \\
\hline
\end{tabular}


Table 7 Comparison of the uses of all common species inventoried in Table 6 to worldwide uses

\begin{tabular}{|c|c|c|c|c|c|c|c|}
\hline Scientific name & Present study & $\begin{array}{l}\text { Rabearivony } \\
\text { et al. [7] }\end{array}$ & $\begin{array}{l}\text { Rakotonandrasana } \\
\text { [39] }\end{array}$ & $\begin{array}{l}\text { Razafindraibe } \\
\text { et al. [8] }\end{array}$ & Quansah [19] & Nicolas [38] & Worldwide \\
\hline $\begin{array}{l}\text { Aeschynomene } \\
\text { sensitiva Sw. }\end{array}$ & Malaria & & & & & & \\
\hline $\begin{array}{l}\text { Aframomum } \\
\text { angustifolium } \\
\text { (Sonn.) K. Schum. }\end{array}$ & Cough & & & & & Cough & \\
\hline $\begin{array}{l}\text { Aphloia theiformis } \\
\text { (Vahl) Benn. }\end{array}$ & Stomach-ache & & & Malaria & & Malaria & \\
\hline $\begin{array}{l}\text { Artocarpus } \\
\text { heterophyllus Lam. }\end{array}$ & Diarrhea & & & & & Diarrhea & Diarrhea $[43,44]$ \\
\hline Canarium L. & Diarrhea & & & & & & \\
\hline $\begin{array}{l}\text { Catharanthus } \\
\text { roseus (L.) G. Don }\end{array}$ & Malaria & Stomach-ache & & Stomach-ache & & & $\begin{array}{l}\text { Malaria [45], } \\
\text { diarrhea, } \\
\text { dysentery [46], } \\
\text { diarrhea [44] }\end{array}$ \\
\hline Citrus aurantium L. & Stomach-ache & Cough & & Cough, malaria & & Cough & Diarrhea [44] \\
\hline $\begin{array}{l}\text { Citrus limon (L.) } \\
\text { Burm. f. }\end{array}$ & Cough & & & & & & $\begin{array}{l}\text { Malaria [47], } \\
\text { dysentery [48] }\end{array}$ \\
\hline $\begin{array}{l}\text { Clidemia hirta (L.) } \\
\text { D. Don }\end{array}$ & $\begin{array}{l}\text { Diarrhea, } \\
\text { malaria, } \\
\text { stomach-ache }\end{array}$ & Stomach-ache & & & & & \\
\hline Curcuma longa $\mathrm{L}$. & Stomach-ache & Malaria & & Malaria & & & Cough [49] \\
\hline $\begin{array}{l}\text { Cyanthillium } \\
\text { cinereum (L.) H. Rob. }\end{array}$ & Stomach-ache & & & & & & \\
\hline $\begin{array}{l}\text { Danais terminalis } \\
\text { Boivin ex Drake }\end{array}$ & Diarrhea & & & & & & \\
\hline $\begin{array}{l}\text { Desmodium } \\
\text { ramosissimum } \\
\text { G. Don }\end{array}$ & Cough, diarrhea & Diarrhea & & & & & \\
\hline $\begin{array}{l}\text { Elephantopus } \\
\text { scaber L. }\end{array}$ & $\begin{array}{l}\text { Diarrhea, } \\
\text { stomach-ache }\end{array}$ & & Diarrhea & & Dysentery & & $\begin{array}{l}\text { Diarrhea, } \\
\text { dysentery [43] }\end{array}$ \\
\hline $\begin{array}{l}\text { Entada gigas (L.) } \\
\text { Fawc. \& Rendle }\end{array}$ & Diarrhea & Diarrhea & & & & & \\
\hline $\begin{array}{l}\text { Eriobotrya japonica } \\
\text { (Thunb.) Lindl. }\end{array}$ & Cough & Bilharzia & & & & & Cough [50] \\
\hline $\begin{array}{l}\text { Exacum } \\
\text { quinquenervium } \\
\text { Griseb. }\end{array}$ & Diarrhea & Malaria & & & & & \\
\hline Ficus polita Vahl & Stomach-ache & Stomach-ache & & & & & \\
\hline $\begin{array}{l}\text { Harungana } \\
\text { madagascariensis } \\
\text { Lam. ex Poir. }\end{array}$ & Diarrhea & Diarrhea & & Diarrhea & & & Malaria $[47,51]$ \\
\hline $\begin{array}{l}\text { Kalanchoe prolifera } \\
\text { (Bowie ex Hook.) } \\
\text { Raym.-Hamet }\end{array}$ & Cough, malaria & Cough & & Cough & & & \\
\hline Lantana camara L. & $\begin{array}{l}\text { Diarrhea, } \\
\text { stomach-ache }\end{array}$ & Malaria & & & & & $\begin{array}{l}\text { Dysentery [43], } \\
\text { cough [52], } \\
\text { malaria [51] }\end{array}$ \\
\hline Litchi chinensis Sonn. & $\begin{array}{l}\text { Diarrhea, } \\
\text { dysentery, } \\
\text { stomach-ache }\end{array}$ & & & Diarrhea & & $\begin{array}{l}\text { Bilharzia, } \\
\text { diarrhea }\end{array}$ & \\
\hline $\begin{array}{l}\text { Lygodium } \\
\text { lanceolatum Desv. }\end{array}$ & Stomach-ache & Diarrhea & & & Stomach-ache & & \\
\hline
\end{tabular}


Table 7 Comparison of the uses of all common species inventoried in Table 6 to worldwide uses (Continued)

\begin{tabular}{|c|c|c|c|c|c|c|c|}
\hline $\begin{array}{l}\text { Macaranga obovata } \\
\text { Boivin ex Baill. }\end{array}$ & Diarrhea & & & & & & \\
\hline $\begin{array}{l}\text { Maesa lanceolata } \\
\text { Forssk. }\end{array}$ & Diarrhea & & & & & & \\
\hline $\begin{array}{l}\text { Manihot esculenta } \\
\text { Crantz }\end{array}$ & Diarrhea & & & & & & Diarrhea [52] \\
\hline $\begin{array}{l}\text { Mauloutchia } \\
\text { humblotii (H. Perrier) } \\
\text { Capuron }\end{array}$ & Cough & Cough & & & & & \\
\hline $\begin{array}{l}\text { Mollugo nudicaulis } \\
\text { Lam. }\end{array}$ & $\begin{array}{l}\text { Malaria, } \\
\text { stomach-ache }\end{array}$ & $\begin{array}{l}\text { Cough, } \\
\text { diarrhea, } \\
\text { malaria }\end{array}$ & & $\begin{array}{l}\text { Cough, } \\
\text { diarrhea, } \\
\text { malaria }\end{array}$ & & & \\
\hline Musa paradisiaca L. & $\begin{array}{l}\text { Diarrhea, } \\
\text { dysentery }\end{array}$ & Diarrhea & & Diarrhea & & Diarrhea & $\begin{array}{l}\text { Diarrhea, } \\
\text { dysentery [36, 53], } \\
\text { diarrhea [54], } \\
\text { malaria [47], } \\
\text { cough, diarrhea } \\
\text { [52] }\end{array}$ \\
\hline Oxalis corniculata L. & Cough & & & & & & $\begin{array}{l}\text { Diarrhea, } \\
\text { dysentery [36] }\end{array}$ \\
\hline $\begin{array}{l}\text { Paederia thouarsiana } \\
\text { Baill. }\end{array}$ & Stomach-ache & & & & & & \\
\hline $\begin{array}{l}\text { Petchia erythrocarpa } \\
\text { (Vatke) Leeuwenb. }\end{array}$ & Malaria & & & Malaria & & & \\
\hline $\begin{array}{l}\text { Psidium cattleyanum } \\
\text { Sabine }\end{array}$ & Diarrhea & Diarrhea & & Diarrhea & & & \\
\hline Psidium guajava $\mathrm{L}$. & $\begin{array}{l}\text { Diarrhea, } \\
\text { dysentery }\end{array}$ & & & $\begin{array}{l}\text { Diarrhea, } \\
\text { dysentery, } \\
\text { malaria }\end{array}$ & Diarrhea, dysentery & $\begin{array}{l}\text { Cough, } \\
\text { diarrhea, } \\
\text { dysentery, } \\
\text { malaria }\end{array}$ & $\begin{array}{l}\text { Dysentery [36], } \\
\text { diarrhea, } \\
\text { dysentery } \\
{[43,53,55],} \\
\text { diarrhea [56, 57], } \\
\text { cough, diarrhea } \\
\text { [46], diarrhea, } \\
\text { stomach-ache } \\
\text { [50], dysentery, } \\
\text { stomach-ache [52] }\end{array}$ \\
\hline $\begin{array}{l}\text { Raphia farinifera } \\
\text { (Gaertn.) Hyl. }\end{array}$ & Diarrhea & & & & & & \\
\hline $\begin{array}{l}\text { Ravenala } \\
\text { madagascariensis } \\
\text { Sonn. }\end{array}$ & Stomach-ache & $\begin{array}{l}\text { Cough, } \\
\text { stomach-ache }\end{array}$ & & & & & \\
\hline Senna alata (L.) Roxb. & Bilharzia & & & & & & Diarrhea [44] \\
\hline $\begin{array}{l}\text { Sorindeia } \\
\text { madagascariensis } \\
\text { DC. }\end{array}$ & $\begin{array}{l}\text { Diarrhea, } \\
\text { stomach-ache }\end{array}$ & Stomach-ache & Stomach-ache & & Diarrhea & & \\
\hline $\begin{array}{l}\text { Toddalia asiatica (L.) } \\
\text { Lam. }\end{array}$ & $\begin{array}{l}\text { Malaria, } \\
\text { stomach-ache }\end{array}$ & Stomach-ache & & & & & $\begin{array}{l}\text { Diarrhea }[56] \\
\text { malaria }[45,51]\end{array}$ \\
\hline Zea mays $\mathrm{L}$. & Stomach-ache & & & & & Cough & \\
\hline
\end{tabular}

Regarding the uses of plant species recorded, those of the common species reported from the different literature cited in Table 6, including the 16 most relevant species identified in this study, were compared to other uses found in some worldwide literature consulted (Table 7). The table shows that uses are most common around the world for some cosmopolitan species like Artocarpus heterophyllus, Elephantopus scaber, Musa paradisiaca and Psidium guajava. Common use of these plants might indicate their efficacy for treatment. However, our study reported the unique use of eight of the most relevant plant species, of which four (50\%) were endemic to Madagascar. Aeschynomene sensitiva (not endemic) was only used for malaria, Canarium sp. (endemic), Danais terminalis (endemic), Macaranga obovata (endemic), Maesa lanceolata (naturalized) and Raphia farinifera 
(naturalized) for diarrhea, and Cyanthillium cinereum (naturalized) and Paederia thouarsiana (endemic) for stomach-ache. Literature did not report any use of these species for the most frequent diseases. Nevertheless, species within the genus Paederia often have the same use and are generally used for stomach-ache [38].

Currently, no exhaustive list of medicinal plants exists either for Ambalabe or Madagascar in general [39]. Besides, data for different regions and localities are scattered, exist in different formats, and sometimes are hardly accessible [40, 41]. The literature review of Rakotonandrasana [39] reported 2777 medicinal plants recorded in Madagascar, of which $39 \%$ were endemic. Nevertheless, new studies always find new medicinal plants used by Malagasy people. The list increases gradually as new research is done, and still far from complete. Thus, this study largely contributed to the enrichment of data on Malagasy pharmacopeia because research in ethnomedicinal practices can add to the knowledge about new and less known medicinal plants [42].

Many species used medicinally do not occur in the local protected area for which a use management system has been already established. Therefore, most of these species might be threatened due to unsustainable practice. As already discussed by Rabearivony et al. [7], some collecting methods of medicinal plants give cause for conservation concern. As such, suggestions on sustainable harvest and conservation are needed, especially for species that are only found outside protected areas.

\section{Conclusions}

Traditional medicine remains the primary healthcare system in Ambalabe community. Many plant species are used as remedies for multiple ailments. Unfortunately, the use of medicinal plants in Ambalabe community is still not well documented. Based on literature, no previous in depth studies were conducted in this area. This present study was undertaken with the hope of obtaining more detailed information on how medicinal plants in Ambalabe are used, which largely contributed to prevent the loss of knowledge due to ongoing forest destruction.

Our research indicates that the local population retains an important knowledge about medicinal plants used to treat the most frequent diseases. Our first hypothesis was therefore supported. The results also support our second hypothesis, i.e. that many species used for medicinal purposes might be threatened, especially because we could verify that most were not growing in established protected areas.

To conclude, this paper provides new information on medicinal plants used by the local population in Ambalabe community to fight against frequent diseases. Some species seemed new to sciences or sometimes have new uses never recorded. Further pharmacological studies will be needed to better understand the importance of traditional medicine. Besides, because 83 species were used to treat six most frequent diseases, their conservation should be considered as important to ensure sustainable future use, especially due to the fact that most of them were collected in the surroundings of the villages and in non-protected areas. Sustainable management techniques should be considered, especially for Malagasy endangered species.

\section{Additional file}

Additional file 1: Guide d'entretien utilisé. (PDF 15 kb)

\section{Competing interests}

The authors declare that they have no competing interests.

\section{Authors' contributions}

All authors participated in the study design. NHR carried out the study, analyzed the data and drafted the manuscript. All authors read, revised and approved the manuscript.

\section{Acknowledgements}

First, we are thankful to William L. Brown Center at Missouri Botanical Garden for its financial support during the study. We also extend our thanks to the staff of the WLBC Ambalabe project, the local population of the Ambalabe community who contributed in this research, and the local guides for sharing their knowledge.

\section{Author details}

${ }^{1}$ Missouri Botanical Garden, Madagascar Research and Conservation Program, BP 3391, Antananarivo 101, Madagascar. ${ }^{2}$ Department of Plant Biology and Ecology, University of Antananarivo, BP 906, Antananarivo 101, Madagascar. ${ }^{3}$ Department of Anthropology, Washington University, St. Louis, MO 63130, USA. ${ }^{4}$ William L. Brown Center, Missouri Botanical Garden, P.O. Box 299, St. Louis, MO 63166-0299, USA.

Received: 21 May 2015 Accepted: 19 August 2015

Published online: 15 September 2015

\section{References}

1. Seifu T. Ethnobotanical and ethnopharmaceutical studies on medicinal plants of Chifra district, Afar region, North Eastern Ethiopia. PhD Thesis. Addis Ababa University: School of Graduate Studies; 2004.

2. World Health Organisation. Health of indigenous peoples. Geneva, Switzerland: Factsheets N 326; 2007. http://www.who.int/mediacentre/ factsheets/fs326/en/. Accessed 30 April 2015.

3. Bussmann RW, Sharon D. Traditional medicine plant use in Northern Peru: tracking two thousand years of healing culture. J Ethnobiol Ethnomed. 2006;2:47.

4. Bussmann RW, Glenn A. Peruvian medicinal plants for the treatment of liver and gallbladder ailments. Arnaldoa. 2010;17(2):243-53.

5. Bussmann RW, Glenn A. Plants used for the treatment of gastro-intestinal ailments in Northern Peruvian ethnomedicine. Arnaldoa. 2010;17(2):255-70.

6. Randrianarivelojosia M, Rasidimanana VT, Rabarison H, Cheplogoi PK, Ratsimbason M, Mulholland DA, et al. Plants traditionally prescribed to treat tazo (malaria) in the eastern region of Madagascar. Malar J. 2003;2:25.

7. Rabearivony DA, Kuhlman AR, Razafiarison ZL, Raharimalala F, Rakotoarivony F, Randrianarivony $T$, et al. Ethnobotanical study of the medicinal plants known by men in Ambalabe, Madagascar. Ethnobotany Research \& Applications. 2015;14:123-38.

8. Razafindraibe M, Kuhlman AR, Rabarison H, Rakotoarimanana V, Rajeriarison C, Rakotoarivelo N, et al. Medicinal plants used by women from Agnalazaha littoral forest (Southeastern Madagascar). J Ethnobiol Ethnomed. 2013;9:73.

9. Conservation International, World Wildlife Fund. Assessing the impacts of climate change on Madagascar's biodiversity and livelihoods - A workshop report. Antananarivo: MEEFT; 2008. 
10. Green GM, Sussman RW. Deforestation history of the eastern rainforests of Madagascar from satellite images. Science. 1990;248(4952):212-5.

11. Busch J, Dave R, Hannah L, Cameron A, Rasolohery A, Roehrdanz P, et al. Climate change and the cost of conserving species in Madagascar. Conserv Biol. 2012;26(3):408-19.

12. Aubert $S$, Razafiarison S, Bertrand A. Déforestation et systèmes agraires à Madagascar : Les dynamiques des tavy sur la côte orientale. Montpellier: CIRAD; 2003.

13. Gómez-Baggethun E, Mingorría S, Reyes-García V, Calvet L, Montes C. Traditional ecological knowledge trends in the transition to a market economy: empirical study in the Doñana Natural Areas. Conserv Biol. 2010;24(3):721-9.

14. Reyes-García V, Guèzeb M, Luz AC, Paneque-Gálvez J, Macía MJ, Orta-Martínez M, et al. Evidence of traditional knowledge loss among a contemporary indigenous society. Evol Hum Behav. 2013;34(4):249-57.

15. Oliver SJ. The role of traditional medicine practice in primary health care within Aboriginal Australia: a review of the literature. J Ethnobiol Ethnomed. 2013:9:46

16. Badiaga M. Etude ethnobotanique, phytochimique et activités biologiques de Nauclea latifolia Smith une plante médicinale africaine récoltée au Mali. PhD Thesis. University of Bamako: Faculté des Sciences et Techniques (FAST); 2011.

17. Rakotoarivelo N, Razanatsima A, Rakotoarivony F, Rasoaviety L, Ramarosandratana AV, Jeannoda V, et al. Ethnobotanical and economic value of Ravenala madagascariensis Sonn. in Eastern Madagascar. J Ethnobiol Ethnomed. 2014;10:57.

18. Cornet A. Essai cartographique bioclimatique à Madagascar, carte à 1/2 000 000 et notice explicative $N^{\circ}$ 55. Paris: ORSTOM; 1974.

19. Quansah N. Ethnomedicine in the Maroantsetra region of Madagascar. Econ Bot. 1988;42(3):370-5.

20. Styger E, Rakotondramasy HM, Pfeffer MJ, Fernandes ECM, Bates DM. Influence of slash-and-burn farming practices on fallow succession and land degradation in the rainforest region of Madagascar. Agric Ecosyst Environ. 2007;119:257-69.

21. Ravololonanahary H, Rajaspera B. Corridor Ankeniheny-Zahamena : un joyau de I'Ala Atsinanana. In: Conservation International, editor. Songadina $\mathrm{n}^{\circ} 1$. Madagascar: Bulletin trimestriel; 2009. p. 1.

22. Madagascar Catalogue. Catalogue of the Vascular Plants of Madagascar. Missouri Botanical Garden, St. Louis, U.S.A. \& Antananarivo, Madagascar. 2013. http://www.tropicos.org/project/mada. Accessed 9 Jan 2015.

23. Tropicos. Missouri Botanical Garden, St. Louis, U.S.A. 2014. http://www.tropicos.org. Accessed Dec 2014.

24. Alexiades MN, Sheldon JW. Selected Guidelines for Ethnobotanical Research: A Field Manual. Bronx, N.Y., U.S.A: New York Botanical Garden; 1996.

25. Convention on Biological Diversity. Nagoya protocol on access to genetic resources and the fair and equitable sharing of benefits arising from their utilization to the convention on biological diversity. United Nations, Montreal, Canada: Secretariat of the Convention on Biological Diversity; 2011.

26. Martin GJ. Ethnobotany: A Methods Manual. London: Chapman and Hall; 1995.

27. Cámara-Leret R, Paniagua-Zambrana N, Macía MJ. A standard protocol for gathering palm ethnobotanical data and socioeconomic variables across the Tropics. In: Ponman B, Bussmann RW, editors. Medicinal plants and the legacy of Richard E. Schultes. Proceedings of the Botany 2011 Richard E Schultes Symposium. Graficart: Trujillo; 2012. p. 41-71.

28. Borgatti SP. AnthroPac methods guide. Columbia, SC: Analytic Technologies; 1992.

29. Winch PJ, Wagman JA, Malouin RA, Mehl GL. Recherche qualitative pour des programmes de santé améliorés: Guide aux manuels traitant de la recherche participative en matière de santé de l'enfant, de nutrition et de santé reproductive. Afrique: USAID, Division du Développement Durable; 2001.

30. Quinlan M. Considerations for collecting freelists in the field: examples from ethnobotany. Field Methods. 2005;17(3):1-16.

31. Borgatti SP, Halgin DS. Elicitation techniques for cultural domain analysis. In: Schensul J, LeCompte M, editors. Ethnographer's Toolkit. Walnut Creek: Altamira Press; 1999.

32. Friedman J, Yaniv Z, Dafni A, Palewitch D. A preliminary classification of the healing potential of medicinal plants, based on a rational analysis of an ethnopharmacological field survey among Bedouins in the Negev Desert, Israel. J Ethnopharmacol. 1986;16:275-87.

33. Hoffman B, Gallaher T. Importance indices in Ethnobotany. Ethnobotany Research \& Applications. 2007;5:201-18.

34. Medeiros MFT, Silva PS, Albuquerque UP. Quantification in ethnobotanical research: an overview of indices used from 1995 to 2009. Sitientibus série Ciências Biológicas. 2011;11(2):211-30.
35. Andriamparany JN, Brinkmann K, Jeannoda V, Buerkert A. Effects of socio-economic household characteristics on traditional knowledge and usage of wild yams and medicinal plants in the Mahafaly region of south-western Madagascar. J Ethnobiol Ethnomed. 2014;10:82.

36. Laloo D, Hemalatha S. Ethnomedicinal plants used for diarrhea by tribals of Meghalaya Northeast India. Pharmacognosy Review. 2011;5(10):147-54.

37. Gairola S, Sharma J, Gaur RD, Siddiqi TO, Painuli RM. Plants used for treatment of dysentery and diarroea by the Bhoxa community of district Dehradun, Uttarakhand India. J Ethnopharmacol. 2013;150(3):989-1006.

38. Nicolas J-P. Plantes médicinales du Nord de Madagascar - Ethnobotanique Antakarana et informations scientifiques. Madagascar: Jardins du Monde; 2012.

39. Rakotonandrasana SR. Les plantes médicinales de l'aire protégée de Zahamena (Madagascar) et de ses environs : richesse floristique et endémicité. In: Beau N, Dessein S, Robbrecht E, editors. African Plant Diversity: Systematics and Sustainable Development. Proceedings of the XIX th $A E T F A T$ Congress 2010. Madagascar: Scripta Botanica Belgica; 2013;50:356-62.

40. Gaikwad J, Wilson K, Kohen J, Vemulpad S, Jamie J, Ranganathan S. Combining ethnobotany and informatics to discover knowledge from data. In: Rai MK, Acharya D, Rios JL, editors. Ethnomedicinal Plants: Revitalization of traditional knowledge of herbs. New Hampshire: Science Publishers; 2010. p. 444-57.

41. Chavan V, Watve AV, Londhe MS, Rane NS, Pandit AT, Krishnan S. Cataloguing Indian biota: The electronic catalogue of known Indian fauna. Curr Sci. 2004;87:749-63.

42. Joshi M, Kumar M, Bussmann RW. Ethnomedicinal uses of plant resources of the Haigad watershed in Kumaun Himalaya, India. Medicinal and Aromatic Plant Science and Biotechnology. 2010;4(1):43-6.

43. Johnsy G, Beena S, Kaviyarasan V. Ethnobotanical survey of medicinal plants used for the treatment of diarrhea and dysentery. Int J Medicine and Medical Sciences. 2013;3(1):332-8.

44. Das PR, Akter S, Md. Islam T, Kabir MH, Md. Haque M, Khatun Z, Md Nurunnabi, Khatun Z, Lee $Y$, Jahan R, Rahmatullah M. A selection of medicinal plants used for treatment of diarrhea by folk medicinal practitioners of Bangladesh. American-Eurasian J Sustainable Agriculture. 2012, 6(3):153-161

45. Ngarivhume T, van't Klooster CIEA, de Jong JTVM, Van der Westhuizen $\mathrm{JH}$. Medicinal plants used by traditional healers for the treatment of malaria in the Chipinge district in Zimbabwe. J Ethnopharmacol. 2015;159:224-37.

46. Maroyi A. Traditional use of medicinal plants in south-central Zimbabwe: review and perspectives. J Ethnobiol Ethnomed. 2013;9:31.

47. Betti JL, Yongo OD, Obiang Mbomio D, Midoko Iponga D, Ngoye A. An ethnobotanical and floristical study of medicinal plants among the Baka pygmies in the periphery of the Ipassa-Biosphere Reserve Gabon. Eur J Medicinal Plants. 2013;3(2):174-205.

48. Wagh W, Jain AK, Kadel C. Ethnomedicinal plants used for curing dysentery and diarrhoea by tribals of Jhabua district (Madhya Pradesh). Indian J Nat Products and Resources. 2011;2(2):256-60.

49. Punjani BL, Kumar V. Traditional medicinal plant remedies to treat cough and asthmatic disorders in the Aravalli ranges in North Gujarat India. J Nat Remedies. 2002;2(2):173-8.

50. Andrade-Cetto A. Ethnobotanical study of the medicinal plants from Tlanchinol, Hidalgo, México. J Ethnopharmacol. 2009;122:163-71.

51. Bahekar S, Kale R. Herbal plants used for the treatment of malaria - A literature review. J Pharmacognosy and Phytochemistry. 2013;1(6):141-6.

52. Albuquerque UP, Medeiros PM, Almeida ALS, Monteiro JM, Freitas Lins Neto EM, Melo JG, et al. Medicinal plants of the caatinga (semi-arid) vegetation of NE Brazil: A quantitative approach. J Ethnopharmacol. 2007;114:325-54.

53. Gangte HE, Zomi GT, Thoudam NS. Ethnomedicinal plants used in diarrhoea and dysentery by the Zou tribe in Churachandpur district, Manipur, India. Asian J Experimental Biological Sciences. 2013;4(3):369-76.

54. Kidane B, van Andel T, van der Maesen LJG, Asfaw Z. Use and management of traditional medicinal plants by Maale and Ari ethnic communities in southern Ethiopia. J Ethnobiol Ethnomed. 2014;10:46.

55. Kar A, Borthakur SK. Medicinal plants used against dysentery, diarrhoea and cholera by the tribes of erstwhile Kameng district of Arunachal Pradesh. Natural Product Radiance. 2008;7(2):176-81. 
56. Shanmugam S, Annadurai M, Rajendran K. Ethnomedicinal plants used to cure diarrhoea and dysentery in Pachalur hills of Dindigul district in Tamil Nadu, Southern India. Journal of Applied Pharmaceutical Science. 2011;1(8):94-7.

57. Tangjitman K, Wongsawad C, Kamwong K, Sukkho T, Trisonthi C.

Ethnomedicinal plants used for digestive system disorders by the Karen of northern Thailand. J Ethnobiol Ethnomed. 2015;11:27.

Submit your next manuscript to BioMed Central and take full advantage of:

- Convenient online submission

- Thorough peer review

- No space constraints or color figure charges

- Immediate publication on acceptance

- Inclusion in PubMed, CAS, Scopus and Google Scholar

- Research which is freely available for redistribution 